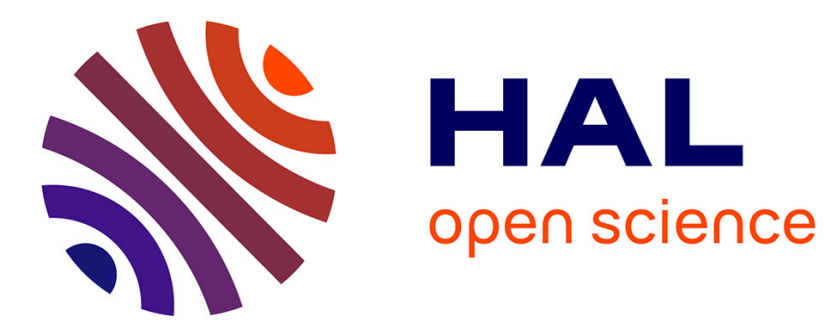

\title{
Petite histoire d'espace et d'identité dans une ville sud-africaine \\ Philippe Gervais-Lambony
}

\section{To cite this version:}

Philippe Gervais-Lambony. Petite histoire d'espace et d'identité dans une ville sud-africaine. Champ Psychosomatique, 2001, 21, pp.119-131. 10.3917/cpsy.021.0119 . hal-02319856

\section{HAL Id: hal-02319856 \\ https://hal.parisnanterre.fr/hal-02319856}

Submitted on 18 Oct 2019

HAL is a multi-disciplinary open access archive for the deposit and dissemination of scientific research documents, whether they are published or not. The documents may come from teaching and research institutions in France or abroad, or from public or private research centers.
L'archive ouverte pluridisciplinaire HAL, est destinée au dépôt et à la diffusion de documents scientifiques de niveau recherche, publiés ou non, émanant des établissements d'enseignement et de recherche français ou étrangers, des laboratoires publics ou privés. 


\title{
Petite histoire d'espace et d'identité dans une ville sud-africaine
}

\author{
Philippe Gervais-Lambony
}

\section{UNE FAMILLE DE L'EAST RAND, AFRIQUE DU SUD}

«

laatjie, en Afrikaans, signifie petit objet ramassé par terre, par exemple un morceau de métal, un caillou, un objet sans valeur... Ce mot, au Xvire siècle, a été utilisé comme nom d'esclave par les maîtres afrikaners » (c'està-dire les descendants des Hollandais installés sur la péninsule du Cap de Bonne Espérance depuis 1652). Cette étymologie est celle que m'indique mon interlocuteur, M. Plaatjies, fier de son ascendance mais qui voit dans son nom une dépréciation humiliante des esclaves par les Blancs. Il semble bien qu'il ait tort pourtant... si le nom de Plaatje était bien jadis donné aux esclaves, il vient en vérité de Platon, comme d'autres esclaves étaient appelés Apollon...

M. Plaatjies vous explique bien volontiers sa version de l'origine de son nom, assis dans le salon de sa maison de Reiger Park, un quartier de la municipalité de Boksburg situé à quelques kilomètres à l'est de Johannesburg, dans la région urbaine de l'East Rand.

La famille Plaatjies est originaire de la région du Cap de l'Est, au sud du pays. L'oncle de M. Plaatjies est venu, avec sa femme et ses enfants, travailler dans l'East Rand en 1935. A cette époque l'East Rand était la première région minière du pays et une des plus importantes du monde: des sociétés minières aux capitaux sud-africains et internationaux y extra-

Philippe Gervais-Lambony - Université de Paris X-Nanterre - Département de géographie 
yaient de l'or en grande quantité. C'est aussi, encore de nos jours, une région industrielle : production chimique, métallurgique et mécanique se sont développées d'abord au service des mines, puis de façon autonome. L'appel de main-d'œuvre dans l'East Rand des années 1930 aux années 1960 fut si considérable que se mit en place une vaste région urbaine, le Witwatersrand, centrée sur Johannesburg mais qui comprend aussi le West et l'East Rand, ensemble de villes minières nées à la fin du xIxe siècle et coalescentes. Aujourd' hui, cet ensemble urbain compte au moins 6 millions d'habitants, dont deux pour l'East Rand.

L'oncle de M. Plaatjies était employé par la New Model Gold Mine, située sur la municipalité de Benoni. Il habitait la "location" d'Actonville (à Benoni, au nord de l'East Rand), c'est-à-dire le quartier réservé aux populations "non-blanches". Depuis le début du siècle, toutes les villes de la région avaient mis en place un ou plusieurs quartiers de ce type. Les "locations" pouvaient être des lotissements municipaux ou privés, elles étaient situées près des centres-villes et habitées par une population relativement mélangée socialement et racialement. Sudafricains d'origine indienne, Noirs, "métis", et même quelques blancs, cohabitaient là... de même que s'y côtoyaient des citadins de niveaux sociaux différents, de l'employé de maison à l'instituteur, du petit commerçant au migrant à la recherche d'un emploi... Certains d'ailleurs étaient propriétaires de leur logement, d'autres locataires. Néanmoins, dans la société sud-africaine de l'époque, les droits civiques des habitants des "locations" étaient limités : leur seule participation à la vie politique de la cité était l'élection d'un Advisory Board, conseil uniquement consultatif qui, sous la houlette d'un administrateur blanc, était censé servir de relais entre la population du quartier, le conseil municipal (composé de Blancs élus par la population blanche) et son administration. Espace faiblement citoyen, la "location" était néanmoins un espace citadin essentiel. Très densément peuplée, et de plus en plus au fur et à mesure que la population du Witwatersrand augmentait, elle servait de centre à la vie sociale, politique et culturelle de tous les "non-Blancs" de la ville, ceux qui vivaient là, mais aussi les mineurs, hommes seuls logés dans des cités fermées, qui fréquentaient le weekend les bars illégaux de la "location", et, dans une moindre mesure, les habitants des bidonvilles qui se développaient aux marges de la ville. Les bars illégaux (les shebeen) résistaient à la prohibition de la production, vente et consommation d'alcool 
par les noirs imposée entre 1928 et 1962 (le seul alcool légal était la bière dont la vente est un monopole municipal). Mais, dans l'illégalité des lieux de vente, maisons anonymes tenues par des femmes (les shebeen queens), se développa une contreculture noire intense.

M. Plaatjies arriva à Actonville en 1951, à l'âge de 20 ans. Il venait de finir ses études dans un collège de mission dans l'Etat Libre d'Orange où il avait obtenu son diplôme d'instituteur. Formation courte, car M. Plaatjies, n'étant pas blanc (on dirait en français que sa peau est "café au lait"), était, selon les principes de l'apartheid, destiné à devenir enseignant pour sa propre communauté : les coloured. En effet, le Parti National, arrivé au pouvoir en 1948, était en train de mettre en place ce que ses idéologues avaient défini comme l'apartheid. En 1950 fut voté le Population Registration Act qui imposa que chaque sud-Africain soit "classé" dans un des quatre groupes raciaux définis par les autorités : Blanc, Bantou, Asiatique et Coloré. Les Bantous sont les sud-Africains noirs, les Asiatiques sont essentiellement composés de sud-Africains d'origine indienne ("importés" en Afrique du Sud par les Britanniques au XIXe siècle pour travailler sur les plantations de canne à sucre), les Blancs sont les sud-Africains d'origine européenne (essentiellement Afrikaners et Anglo-saxons). Les Colorés ? Ce sont les autres : descendants d'esclaves malais, populations aborigènes autochtones, métis d'aborigènes et de Bantous ou de Blancs, de Bantous et de Blancs... C'est donc une définition identitaire négative : appartenaient au groupe des colorés tous les sud-Africains qui ne pouvaient être définis autrement ${ }^{1}$. En 1996, 3,6 millions de sud-Africains se définissent ${ }^{2}$ encore comme colorés, soit presque $9 \%$ de la population totale. De langue maternelle essentiellement afrikaans, cette population est surtout importante dans la province du Cap où elle est majoritaire.

Selon la loi, en 1951, M. Plaatjies était donc coloured. Le développement séparé, prôné par l'apartheid, supposait en théorie que chaque communauté raciale puisse se développer, selon sa culture, indépendamment. Il fallait donc que la vie chaque communauté puisse se dérouler dans un espace à part. C'est pourquoi l'apartheid, par définition, est une politique géographique, au sens où l'espace est utilisé pour modeler la société et imposer des identités à chacun. Ainsi, en Afrique du Sud, les rapports entre espaces et identités ont toujours été forts parce que l'espace y a été sciemment utilisé pour imposer une culture
1. C'est pourquoi il est inexact de traduire le terme coloured par le mot métis, il s'agit d'un groupe complexe, loin d'être composé entièrement de métis.

2. Dans le recensement de 1996, il était demandé aux recensés de se définir eux-mêmes alors que dans les recensements de l'apartheid c'était le recenseur qui cochait la case "race". Seuls 0,9\% des recensés ont refusé de se définir selon ce critère ce qui prouve la prégnance de l'identification raciale. 
dominante (d'origine européenne) et en définir d'autres, dominées (africaines ou plus généralement non-blanches). La méthode était d'imposer une identité à travers une définition territoriale. Cette politique raciste faisait de l'Afrique du Sud un cas unique au moins de deux points de vue : le racisme y était inscrit dans la loi et le partage, très inégal, de l'espace national était strictement fondé, à toutes les échelles, sur de prétendues identités raciales et ethniques.

Au moment ou M. Plaatjies arriva à Actonville, l'arsenal législatif de l'apartheid était en train d'être mis en place. Certes l'apartheid interdisait les unions mixtes et les relations sexuelles inter-raciales et imposait la ségrégation des lieux publics, depuis les plages jusqu' aux bureaux de poste en passant par les transports en commun et les lieux de distraction (Reservation of Separate Amenities Act, 1952). Mais, au-delà de ces règlements de "l'apartheid mesquin" (ou "petit apartheid"), le "grand apartheid" reposait sur la division de l'espace et l'application du Group Areas Act de 1950, qui définissait les zones de résidence de chaque groupe racial et l'organisation du territoire en terres européennes d'une part et réserves africaines d'autre part. Dans le même temps, le Bantu Education Act de 1952 imposait la division raciale des systèmes éducatifs et le port d'un passeport intérieur (le pass) pour les citadins noirs.

On comprend que l'apartheid ne pouvait tolérer les "locations": mal contrôlées, proches de la ville des Blancs, ces quartiers étaient caractérisés par la coexistence de différents groupes raciaux et aussi par l'appropriation foncière par certains nonBlancs. Ce dernier point est essentiel : pour le gouvernement de l'apartheid, les villes étaient des espaces réservés aux Blancs, les Noirs sud-africains ne devaient y être que temporairement, le temps de leur vie active. Ainsi, la propriété noire en ville était contradictoire avec l'apartheid. C'est pourquoi les "locations", qui dataient du début du siècle, voire étaient plus anciennes (les plus célèbres s'appelaient Sophiatown à Johannesburg, Cato Manor à Durban, District Six au Cap, Marabastad à Pretoria) furent toutes rasées dans les années 1950 ou 1960. C'est aussi pourquoi elles restèrent dans les mémoires noires comme une sorte d'idéal urbain. A la place des "locations", le Group Areas Act imposa la construction de quartiers ségrégués entièrement locatifs : les townships. Ce fut aussi l'occasion d'imposer aux "non-Blancs" citadins une identité conforme aux idéaux de l'apartheid. 


\section{LE TOWNSHIP : FORME SPATIALE ET IDENTITÉ}

Le terme township signifie lotissement, mais en Afrique du Sud il désigne seulement les lotissements publics réservés naguère aux non-Blancs (Noirs, Indiens, Colorés). Par extension, le terme désigne aussi une culture, une identité, un mode de vie comme en attestent un certain nombre d'expressions courantes : township life, townwship boy, township music, township driving... Le township est enfin une forme urbaine indissociable des politiques de contrôle des populations noires sous l'apartheid et le reflet direct d'une idéologie.

Le township est un ensemble monotone de maisons, identiques les unes aux autres, alignées le long de rues rectilignes, entouré d'une zone non-bâtie, la buffer zone (zone tampon) qui l'isole du reste de l'espace urbain. L'urbanisme du township combine des considérations d'ordre économique (construire pour pas cher) et des considérations de nature sécuritaire : les zones-tampons permettent l'encerclement du quartier, les axes larges facilitent la circulation des véhicules des forces de sécurité, les hautes tours d'éclairages éclairent de vastes zones et ne sont pas sabotables aisément.

A la construction massive des townships il y a deux ordres de causes directes. D'une part il était nécessaire de construire massivement en ville pour loger une population urbaine noire en accroissement très rapide durant les années 1940, période d'industrialisation rapide et de boom économique (la population africaine de Johannesburg passa de 527507 au recensement de 1936 à 915334 à celui de 1951). D'autre part, le régime qui arrivait au pouvoir en 1948 avait besoin d'une forme urbaine qui lui permette de contrôler la population non-blanche et d'imposer systématiquement la ségrégation en ville.

Le township est pris en charge par le gouvernement qui peut ainsi imposer sa volonté aux autorités locales en échange du logement de la main-d'œuvre. Parc strictement locatif, le township est conforme à l'idéologie qui définit les Africains comme des migrants temporaires en ville. Il permet, par le déplacement des populations, de briser les structures associatives et surtout politiques des anciennes "locations" (dans lesquelles, surtout dans le Witwatersrand, le parti communiste était très solidement implanté). Enfin, en distinguant strictement les budgets des villes et ceux des townships (financés par les loyers et taxes payés par habitants et la vente de bière par les municipalités), 
l'Etat offre une solution économique aux villes pour le logement de la main-d'œuvre. En outre, en interdisant les activités économiques aux Africains dans les townships, le système assure une clientèle captive aux commerces de la ville blanche. Le township est donc l'élément essentiel d'un véritable système urbain qui est à la fois politique, social et économique.

Pour les habitants noirs des villes, les conséquences immédiates du déplacement forcé vers les townships sont l'éloignement, l'augmentation des dépenses de transport et des charges et loyers (et pour les propriétaires l'expropriation). En outre leur vie quotidienne est réglée: les demandes d'autorisation aux autorités du township sont nécessaires pour recevoir des visiteurs, organiser des fêtes, etc.

Du point de vue du régime, un autre élément est essentiel: le township, parce que strictement contrôlé, est le moyen de faire appliquer les lois sur les migrations (influx control). En effet, puisque les maisons sont toutes attribuées par les autorités et ne le sont que pour des actifs employés par l'économie blanche, il n'y a en théorie plus de place pour une population "excédentaire" en ville. Il s'agit bien d'une forme de gestion de la main-d'œuvre. Le modèle est le compound minier : logement en cités fermées de travailleurs sous contrat, c'est pourquoi, par la suite, furent construits dans les townships, de vastes hostels, bâtiments collectifs destinés au logement des travailleurs célibataires, idéal absolu de la ville d'apartheid...

Mais il ne faut pas négliger le fait que le township est aussi un outil de construction identitaire. D'abord il assure la séparation des groupes raciaux tels que définis par l'apartheid en créant aussi des townships propres aux Colorés et aux Indiens. Il impose donc à chacun sa place dans l'espace en fonction de l'appartenance raciale. Ensuite, à l'intérieur des townships noirs sont généralement distingués des secteurs ethniques, deuxième niveau d'identité imposé aux populations noires (de mêmeque dans les mines les travailleurs migrantsétaient répartis par ethnies). En créant ces territoires raciaux et ethniques on impose une identité. Mais le township est aussi construction identitaire par le bas et l'inversion se fait rapidement. Les populations déplacées reconstruisent leur espace et leurcommunauté dans le township, et la société du township finit parêtre aussi forte et dangereuse pour le pouvoir que celle des anciennes "locations". D'espace imposé, le township devient vite espace récupéré : la ségrégation est doublement construction territoriale et identitaire, par le haut et par le bas. 


\section{REIGER PARK : UN QUARTIER IDENTITAIRE}

Dans l'East Rand, l'application du Group Areas Act prit plus de 10 ans. Les négociations entre le pouvoir central et les municipalités furent longues. De plus, les autorités centrales souhaitaient que la répartition raciale des populations se fasse à l'échelle de l'ensemble des huit villes composant l'East Rand. Il fut décidé la mise en place pour l'East Rand de quatre vastes zones pour le logement des Noirs, mais le cas des populations colorées et des populations indiennes était particulier: disséminées dans toutes les "locations" de l'East Rand, on les rassembla dans deux quartiers seulement, Actonville et Reiger Park. C'est Actonville qui fut désigné comme devant devenir le township indien de l'East Rand. Le quartier ne fut donc pas détruit contrairement aux autres "locations", mais les propriétaires furent expropriés, les locataires expulsés et les maisons attribuées à des familles indiennes. Les populations noires furent envoyées vers divers townships et les Colorés se virent attribuer des maisons dans une autre ancienne "location", Stirtonville. Dans ce grand jeu de l'apartheid, on utilisait un groupe pour en déplacer un autre...

M.Plaatjes, qui avait d'abord habité chez son oncle, s'était installé en 1955 dans une maison d'Actonville qu'il avait achetée. Il y vivait avec sa femme est ses enfants tout en enseignant dans une école pour Colorés d'une autre municipalité (Edenvale). En tant qu'instituteur, il appartenait à l'élite intellectuelle du quartier. Il était membre clandestin de l'ANC (African National Congress), parti politique interdit depuis 1961 (après le massacre de Sharpeville en 19603). En 1964, la famille Plaatjies reçut un courrier de la municipalité de Benoni qui leur signifiait leur expropriation et l'attribution d'un logement à Stirtonville. Une réunion de la population colorée fut organisée par les fonctionnaires municipaux: "you must move" dirent-ils. Certains habitants d'Actonville étaient là depuis plus d'une génération, mais c'était une époque de peur et il n'y eut pas de véritable résistance. M. Plaatjies décida de ne pas partir avec les camions de la police qui vinrent ramasser les gens et leurs biens... Il attendit jusqu'au jour où se présenta devant sa porte une famille indienne avec son déménagement pour prendre possession de la maison. C'était un vendredi après-midi... En une journée le déménagement fut organisé, grâce à l'aide d'un ami qui possédait une camionnette et qui emmena les Plaatjies à Stirton-
3. Lors d'une manifestation au sud de Johannesburg organisée par le Pan African Congress contre le port du passeport intérieur, la police tira sur la foule tuant 59 personnes. Cet événement a provoqué la prise de conscience de l'impasse de la voie légale de lutte contre l'apartheid et provoqué l'entrée en clandestinité des formations d'opposition. En 1964 les principaux leaders de l'ANC sont condamnés à la prison à perpétuité et envoyés à Robben Island (au large du Cap). Nelson Mandela faisait partie de ces leaders, ceux qui échappèrent à l'arrestation s'exilèrent. 
ville. Mais, arrivant le samedi, la famille trouva les bureaux de la municipalité fermés, c'est un ancien voisin qui leur indiqua une maison inoccupée où il s'installèrent.

Stirtonville était un quartier moins bien équipé qu'Actonville. En effet, alors qu' Actonville était un quartier privé, Stirtonville était une ancienne "location" municipale pour Noirs : les maisons sont plus petites, construites au moindre coût... En outre les nouveaux voisins ne se connaissaient pas, ils s'identifiaient comme originaires de Benoni, Germiston, Boksburg, Alberton, etc (noms des différentes villes de l'East Rand d'où ils venaient). Les réseaux associatifs et politiques étaient démantelées. Des gangs de jeunes se mirent en place sur la base de l'origine des groupes, et le temps fut d'abord à la violence, à l'inquiétude et à la micro-territorialisation par quartier d'origine. C'est que, ce qui prime encore, n'est pas l'identité "colorée", mais les anciennes identités des "locations"... D'après M. Plaatjies, c'est au bout de deux ans que se développa un nouveau sentiment communautaire, notamment sous l'impulsion des responsables des églises.

Dans le même temps, les autorités tentaient aussi de construire l'identité du quartier. En 1968 fut mis en place un Advisory Commitee: héritier des advisory board des "locations", ses membres étaient désignés par le conseil municipal blanc parmi les membres d'un parti coloré pro-apartheid.En 1968 furent créées les trois écoles primaires du quartier que la municipalité baptisa respectivement Reiger, Dromaderi et Goede Hoop et l'école secondaire appelée Reiger.Enfin, en 1969, le Conseil municipal de Boksburg débaptisa Stirtonville qui s'appela désormais Reiger Park.Le Reiger, le Dromaderis et le Goede Hoopétaient les trois navires de l'expédition de Jan van Riebeck qui créa la colonie du Cap en 1652. La dénomination des lieux étaient un message clair, qui fut d'ailleurs expliqué aux résidents : vous êtes liés aux Afrikaners, vous êtes originaires de la région du Cap, vous êtes différents des Bantous. Par le cantonnement dans un quartier "ethnique" et sa dénomination, les autorités visaient à fonder une identité "colorée", par delà la diversité sociale de la communauté. C'était aussi diviser pour régner...

Pendant ce temps, les anciens habitants noirs de Stirtonville avaient été déplacés vers le nouveau township de Vosloorus, à une dizaine de kilomètres au sud la ville de Boksburg. Là, selon le même principe, ils avaient été répartis par ethnies entre la section Sotho et la section Nguni... 
Mais, malgré le travail policier et l'emploi d'espions pour dénoncer les activités illégales, une conscience politique différente se mit en place à Reiger Park. Elle trouva son terreau dans des mécontentements très concrets des habitants : coût des loyers trop élevés, manque de maisons, manque de commerces... Les seuls commerces de Reiger Park étaient tenus par des Indiens (qui étaient déjà les seuls commerçants de la "location" de Stirtonville) car les Colorés n'avaient pas le droit de tenir des boutiques. En 1975, une émeute donna lieu au pillage des commerces indiens. Un temps de tension s'ensuivit, d'autant plus qu'en juin 1976 les "émeutes de Soweto" déclenchèrent des troubles considérables dans tous les quartiers noirs. Finalement le Conseil Municipal autorisa l'installation de Colorés dans le quartier voisin de Windmill Park. Quelques blancs y résidaient que l'on ne voulu pas expulser : le quartier fut donc déclaré "zone grise", c'est-à-dire qu'une occupation multiraciale y était autorisée. Des Noirs vinrent aussi s'installer, alors que les blancs quittaient le quartier: un espace assez similaire aux anciennes "locations" se reconstitua donc... En outre on construisit de nouveaux logements à Reiger Park : des maisons plus grandes et mises en vente, c'est dans l'une d'elle que la famille Plaatjies s'installa en 1984.

Durant les années 1980, le gouvernement multiplia les signes en directions des communautés colorées et indiennes. Le plus fort fut la modification de la constitution qui instituait trois parlements : un blanc, un coloré, un indien. L'idée du régime était de se trouver des alliés, mais les élections connurent des taux de participation très faibles, et seuls des partis ethniques très minoritaires participèrent. En 1986, le gouvernement abolit les lois sur l'influx control et le "petit apartheid" commença à être démantelé en pratique. Dans le même temps on réprimait violemment les activités politiques dans les townships noirs. Elles y demeurèrent cependant intenses, notamment sous l'impulsion des civics, associations de citadins au niveau local qui mobilisaient les populations et quadrillaient les quartiers. En outre la situation se compliqua par le développement des bidonvilles illégaux, de plus en plus tolérés par les autorités.

Mais, à Boksburg, l'événement important de la fin des années 1980 fut la prise de contrôle du Conseil municipal par le Conservative Party lors des élections locales de 1988. Le CP est à la droite du parti national, il fit campagne sur le retour aux principes de l'apartheid des années 1950. A Boksburg, le CP 
décida d'appliquer le Separate Amenities Act de 1953: toilettes, bancs publics, bus... tout fut re-ségrégué. Très symboliquement, la rue qui entoure la promenade du bord du petit lac de Boksburg, avec ses aires de pique-nique et ses pédalos, fut grillagée : l'usage du lieu était réservé aux Blancs. C'est de Reiger Park que fut lancé par les civics un mouvement de protestation dont M. Plaatjies fut un des animateurs, mais il fut lancé en association avec les civics noirs du township de Vosloorus : il s'agissait d'une campagne de boycott des commerces de la ville blanche de Boksburg. Les citadins organisèrent un système de bus pour aller faire les courses dans les municipalités voisines. Sous la pression des commerçants, la municipalité dut céder et les conseillers $\mathrm{CP}$ démissionnèrent les uns après les autres. Le succès du mouvement est du à l'alliance entre les différentes communautés qui transcendent des prétendues identités raciales imposées. En 1993 le Conseil fut repris par le Parti National.

\section{ET MAINTENANT?}

M. Plaatjies est un membre influent de sa communauté. Depuis la fin de l'apartheid et l'élection de Neslon Mandela en 1994, il continue ses activités dans l'ANC et les associations locales. Aux élections municipales de décembre 2000, il sera candidat dans sa circonscription, mais celle-ci ne correspond plus au seul quartier de Reiger Park.

Comment le nouveau gouvernement gère-t-il le lourd héritage urbain de l'apartheid : ville spatialement éclatée, sous-équipement, identités locales très fortes, ségrégation qui se pérennise ? C'est une nouvelle ville qu'il faut créer, fondée sur une nouvelle identité citadine qui doit tendre à s'imposer sur les identités ethniques et territoriales anciennes. Certes on s'occupe de construire des logements et d'équiper les quartiers défavorisés. Mais sur le plan des identités, la voie du changement est celle de la réforme du gouvernement local. Et pour ce faire on utilise à nouveau l'outil spatial : l'apartheid avait employé le découpage de l'espace pour imposer des identités, le nouveau pouvoir souhaite utiliser les découpages territoriaux pour intégrer et créer une identité commune. Les municipalités sont réunifiées, il n'y a plus qu'une seule ville (ce qui permet aussi la redistribution économique). Dans le cas de l'East Rand, c'est 
une autorité métropolitaine qui est créée et qui fédère toutes les anciennes municipalités.

Reiger Park est toujours un quartier, mais il fait partie d'une circonscription qui englobe un bidonville voisin habité par des populations noires et une petite portion de quartier blanc situé au nord. Dans toutes les réunions désormais ces différents groupes se rencontrent, on sent dans ces assemblées un mélange de tension et de volonté de communiquer. Ces différents citadins ne parlent pas la même langue (les habitants du bidonvilles parlent Xhosa, langue du Sud du pays), ils n'ont pas les mêmes intérêts, ils n'ont pas la même couleur de peau, ils n'habitent pas les mêmes quartiers. Il est bien trop tôt pour dire les effets de cette politique, de nouvelles identités vont-elles émerger au niveau des circonscriptions ? Verra-t-on ré-émerger les identités des anciennes municipalités ? Des quartiers quand l'identité de "non-blanc" n'est plus assez fédératrice ? Ou même est-ce que les identités raciales vont être récupérées par des partis politiques "ethniques" ? C'est l'objet de recherches en cours. Je voulais seulement raconter ici une petite histoire d'espace et d'identité avec le regard du géographe. L'apartheid est scientifiquement important en ce sens qu'il peut servir de paradigme de la ségrégation spatiale. En effet, l'histoire de l'Afrique du Sud depuis le XVIIe siècle est comme une longue suite d'avatars de la définition de formes ségrégatives et identitaires. L'apartheid n'en est qu'une version particulièrement aboutie, il existe et a existé en fait dans bien des lieux, ne serait-ce que comme image, modèle ou repoussoir. Au-delà, quelle ville du monde aujourd'hui peut se vanter de ne pas connaître de ségrégation spatiale? Et quelle ségrégation spatiale n'a pas des conséquences identitaires? L'analyse des constructions identitaires (car les identités dont nous parlons ne sont que cela, des construits) par le biais des constructions spatiales sont un des champs de la géographie, par là cette science humaine a son mot à dire sur 1'“identité".
4. Par exemple, à

Johannesburg, en 1997 , des émeutes ont secoué certains quartiers coloured. Les citadins protestaient à l'origine contre des coupures d'eau dans leur quartier et se plaignaient qu'on ne leur accordait pas le même traitement (annulation de dettes et bas tarifs) qu'aux habitants noirs des quartiers voisins : le mouvement fut récupéré sans peine par un parti politique qui revendique la défense des populations coloured. Dans le même ordre d'idée on a constaté lors de toutes les élections depuis 1994 une large part de la population Colorée votait pour le Parti National... 


\section{BIBLIOGRAPHIE}

\section{Afrique du Sud}

CHRISTOPHER A. The Atlas of Apartheid, Johannesburg, Witwatersrand University Press, 1994.

GERVAIS-LAMBONY P., JAGLIN S., MABINA. (éds) La Question urbaine en Afrique australe. Perspectives de Recherche, Paris, Karthala-IFAS, 1999.

GERVAIS-LAMBONY P. Nouvelle Afrique du Sud, nouveaux territoires, nouvelles identités, in l'Espace géographique, ${ }^{\circ} 2$ 2, 1999, pp. 99-109.

GERVAIS-LAMBONY P. L'Afrique du Sud et les Etats voisins, Paris, Armand Colin, collection U Géographie, 1997.

GERVAIS-LAMBONY P. Les villes d'Afrique du Sud : gestion de l'héritage et recomposition de l'espace in Hérodote $n^{\circ}{ }^{82} / 83$, La Découverte, Paris, 1996, pp. 42-60.

GERVAIS-LAMBONY P. (éd) L'Afrique du Sud recomposée, Géographie et Cultures, $\mathrm{n}^{\circ} 28,1998$, Paris, L’Harmattan.

HOUSSAY-HOLZSCHUH M. Le cap, ville sud-africain. Ville blanche, vies noires, Paris, L'Harmattan, 1999.

MEILLASSOUX C. et MESSIANT C. (éds), Génie social et manipulations culturelles en Afrique du Sud, Paris, 1991, Arcantère, pp. 125-143.

\section{Géographie et analyse des identités territoriales}

BERQUE A. Etre humains sur la terre, Paris, Gallimard, 1996.

BRUN J. Essai critique sur la notion de ségrégation et son usage en géographie, in Brun Jacques et Rhein Catherine (éds), La ségrégation dans la ville, Paris, L'Harmattan, 1994, pp. 21-58.

CLAVAL P. Espace et pouvoir, Paris, P.U.F, 1979.

DI MEO G. Géographie sociale et territoires, Paris, Nathan, 1998.

FOUCHER M. Fronts et frontières, un tour du monde géopolitique, Paris, Fayard, 2nde édution, 1991.

RAFFESTIN C. Pour une géographie du pouvoir, Paris, Litec, 1980.

RONCAYOLO M. La ville et ses territoires, Paris, Gallimard (folio), 1990.

\section{RÉSUMÉ}

La géographie humaine a-t-elle quelque chose à dire sur l'identité? Son objet est l'étude des relations entre les hommes, individus ou groupes, et leurs lieux. L'hypothèse est que les hommes et les lieux sont en connivence, en ce sens qu'ils interagissent et constituent des sujets collectifs. Dans cette construction, qui est une construction identitaire, jouent trois éléments : la forme des lieux (matérialité), leur substance (les hommes qui habitent et les relations entre ces hommes), la politique (les actions menées pour modifier forme et substance). C'est l'interaction entre ces trois éléments que le texte veut analyser, très simplement, à travers l'exemple d'un homme et d'un lieu, en Afrique du Sud, peut-être un des pays du monde où les liens entre lieux et iden- 
tités sont les plus forts, en tous cas ont été le plus fortement manipulés par les pouvoirs politiques.

Mots-clés: identité - apartheid - Afrique du Sud - ségrégation - géographie - espace

\section{SUMMARY}

Does human geography have something to say about identity?

Its principal subject is the study of relations between humans, individuals and groups, and their places. Our hypothesis is that humans and places are in complicity in the way they interact and form collective subjects. Three elements come into play in this construction which in a construction of identity: The form of the place (materiality), their substance (the humans who live there and the relations between them), and politics (actions intended to change form and substance). The text wants to analyse the interaction between these three elements very simply following the example of a man and a place in South Africa, possibly one the world's contries where relations between places and identities are among the strongest, where they have been strongly manipulated by political powers in any case.

Key-words: identity - apartheid - South Africa - segregation - geography - space 